\title{
INFLUÊNCIA DA GRANULOMETRIA DO SOLO NA ESTRUTURA DE GUILDAS FUNCIONAIS DE BESOUROS ROLA-BOSTAS (Coleoptera: Scarabaeidae: Scarabaeinae) EM FLORESTAS SEMIDECIDUAIS NO ESTADO DO MATO GROSSO, BRASIL
}

\author{
INFLUENCE OF SOIL GRANULOMETREY ON THE STRUCTURE OF \\ FUNCTIONAL GUILDS OF DUNG BEETLES (Coleoptera: Scarabaeidae: \\ Scarabaeinae) AT SEMIDECIUOUS FORESTS IN THE STATE OF MATO GROSSO, \\ BRAZIL
}

\begin{abstract}
Ricardo J. SILVA ${ }^{1-2}$; Higor V. RIBEIRO ${ }^{3}$; Mateus F. SOUZA ${ }^{4}$; Fernando Z. VAZ-DE-MELLO ${ }^{5-6}$

1. Pós Graduação em Entomologia e Conservação da Biodiversidade, Universidade Federal da Grande Dourados. Rodovia Dourados Itahum, Dourados, MS, Brasil. ricardojosesilva11@gmail.com; 2. Centro de Pesquisa, estudos e desenvolvimento agro-ambiental, Universidade do Estado de Mato Grosso, Tangará da Serra, MT, Brasil; 3. Universidade do Estado de Mato Grosso, Pós Graduação em Ambientes e Sistemas de Produção, Tangará da Serra, MT, Brasil; 4. Pós-graduação em Ecologia e conservação da Biodiversidade, Universidade Federal de Mato Grosso, Cuiabá, MT, Brasil; 5. Departamento de Biologia e Zoologia, Instituto de Biociências, Universidade Federal de Mato Grosso, Cuiabá, MT, Brasil; 6. Muséum National d'Histoire Naturelle, UMR 7205, Département Systématique et Évolution, Entomologie, Paris, France.
\end{abstract}

RESUMO: O objetivo deste trabalho foi descrever as guildas funcionais dos besouros rola-bostas (Scarabaeidae: Scarabaeinae) e analisar a influência da granulometria do solo na estruturação destas guildas e inferir possíveis processos geradores. Para isso, foram coletados besouros rola-bostas e amostras de solo, em áreas de floresta semidecidual, na região norte (módulos do PPbio) e sudoeste (22 reservas de fazendas) do estado de Mato Grosso. Para as coletas dos besouros foram utilizados 85 armadilhas pitfall, iscado com fezes humanas expostas por 48 horas, na região norte e 132 armadilhas na região sudoeste. As duas regiões apresentaram estrutura de guilda similar, com dominância de paracoprídeos, seguido de telecoprídeos e endocoprídeos. Padrão coerente com o padrão Neotropical, o que reforça a teoria da existência de uma estrutura determinística taxonômica e funcional para as subamostras na região. A concentração de argila apresentou efeitos distintos para ambas as regiões estudadas. Na região norte causou redução na abundância de endocoprídeos e telecoprídeos pequenos e reduziu a riqueza de paracoprídeos grandes, enquanto na região sudoeste influenciou principalmente a redução de paracoprídeos pequenos e aumento de telecoprídeos pequenos e endocoprídeos grandes. A concentração de argila no solo influenciou a estrutura das guildas funcionais apenas em nível de micro-habitat. As guildas dos paracoprídeos e telecoprídeos pequenos são as mais afetadas pela variação da granulometria. $\mathrm{O}$ aumento da concentração de argila levou a redução dos paracoprídeos pequenos e aumento dos telecoprídeos pequenos.

PALAVRAS-CHAVE: Solos Amazônicos. Competição inter e intraespecífica. Besouros copro-necrófagos. Escarabeídeos.

\section{INTRODUÇÃO}

Os besouros da subfamília Scarabaeinae são conhecidos como "rola-bostas" pelo hábito característico de alocar excremento, principalmente fezes de mamíferos, e confeccionar bolas com este substrato para serem utilizadas como ninho de abrigo para suas proles (HALFFTER; MATTHEWS, 1966; PHILIPS, 2011). A característica de alocação do recurso permite agrupa-los em três principais grupos: os telecoprídeos que modelam bolas de excremento, rolam e enterram distante da fonte do recurso; os paracoprídeos, que enterram o recurso alimentar em galerias do lado ou sob o recurso; e os endocoprídeos, que nidificam na massa fecal ou sob ela (HALFFTER; MATTHEWS, 1966; HALFFTER; EDMONDS, 1982). Esses grupos de espécies podem ser definidos como guildas funcionais pela similaridade na forma de alocação do recurso (LOUZADA; LOPES, 1997).

Doube (1990) descreve uma estrutura hierárquica competitiva entre os besouros rolabostas e os dividem em dominantes e subordinados. Esta classificação pondera o tamanho da espécie, a maneira e a velocidade de alocação do recurso. Telecoprídeos grandes e pequenos e os paracoprídeos rápidos são considerados competidores dominantes, enquanto paracoprídeos grandes e lentos, paracoprídeos pequenos e lentos, cleptoparasitas e os endocoprídeos são tidos como competidores subordinados. 
Essa classificação possibilita descrever com melhor clareza a estrutura funcional dos besouros rola-bostas, porém apresenta alguns problemas de subjetividade principalmente relacionados à classificação da velocidade de processamento e enterro do recurso. Cleptoparasitas são espécies que depositam seus ovos em bolas-ninhos de outras espécies (HALFFTER; MATTHES, 1966). Essa guilda é de difícil caracterização, pois muitas espécies de pequenos paracoprídeos, como as do gênero Uroxys, são cleptoparasitas facultativos dependendo das condições de disponibilidade de recurso (YOUNG, 2009).

A estrutura da guilda funcional dos besouros rola-bostas para região Neotropical, de maneira geral, está relacionada a fatores históricos e reflete padrões de dominância taxonômica biogeográfica (HALFFTER et al., 1992, LOUZADA; LOPES, 1997; VIDAURRE-SANCHEZ , 2011). Porém fatores locais, formadores do micro-habitat, como tipo de vegetação, umidade e granulometria do solo também são importantes na estruturação das guildas funcionais destes besouros (DOUBE, 1991). O tipo de solo é um fator determinante para a ocorrência de várias espécies de besouros rola-bostas (LUMARET; KIRK, 1991). Os trabalhos realizados no Brasil, em sua maioria, apenas descrevem o padrão geral das proporções de cada guilda e não trazem inferência sobre processos que os geraram (LOUZADA; LOPES, 1997; SILVA et al., 2008; FILGUEIRAS et al., 2011; SILVA; DI MARE, 2012; SILVA et al., 2012). Com isso, este trabalho teve como objetivo descrever as guildas funcionais dos besouros rola-bostas e analisar a influência da granulometria do solo na estruturação destas guildas e inferir possíveis processos geradores destes padrões.

\section{MATERIAL E MÉTODOS}

\section{Área de estudo}

Os estudos foram realizados no município de Cláudia nos módulo do PPbio, $11^{\circ} 54^{\prime} \mathrm{S} ; 55^{\circ} 17^{\prime}$ W (região norte) e nos munícios de Tangará da Serra, Araputanga, Mirassol d'Oeste e Indiavaí entre as coordenadas $14^{\circ} 18^{\prime} \mathrm{S} ; 57^{\circ} 29^{\prime} \mathrm{W}$ e $15^{\circ} 38^{\prime} \mathrm{S}$; $57^{\circ} 54^{\prime}$ W (região sudoeste) (Figura 1).

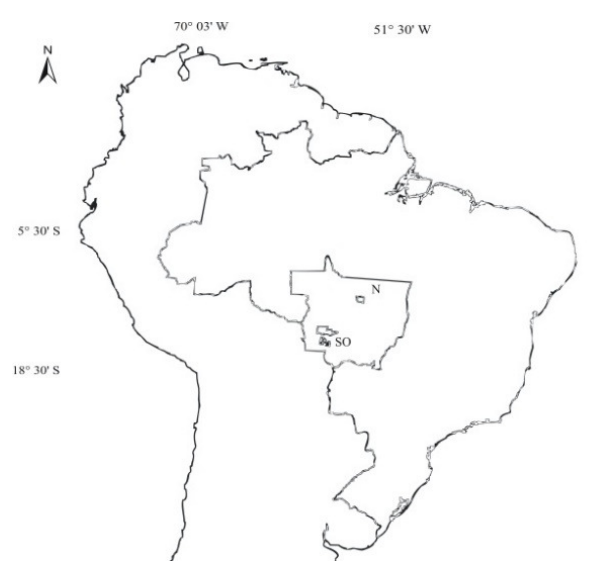

Figura 1. Localização das áreas de estudo no Norte (N) e Sudoeste (SO) do estado de Mato Grosso, Brasil.

A região norte é composta por florestas estacionais semideciduais, limitada ao norte e a oeste pela floresta ombrófila úmida e ao leste e sul pelo cerrado (PPBIO; SENBAM, 2013). O solo é caracterizado como latossolo vermelho - escuro distrófico (SEPLAN, 2001). A proporção de argila variou de 171 a 508 g. $\mathrm{kg}^{-1}$ de solo. Na região sudoeste do estado a vegetação é formada por floresta estacional semidecidual submontana (BRASIL, 1982), e os solos são considerados eutróficos (SEPLAN, 2001). Em grande parte, a depressão do Alto Paraguai é formado por latossolos vermelho-amarelos de constituição argilo-arenosa (SÁNCHEZ, 1992). A proporção de argila variou de
134 a 684 g. $\mathrm{kg}^{-1}$ de solo. De acordo com a classificação climática de Köppen, as regiões de estudos se enquadram no tipo climático Aw, pertencente ao domínio de clima tropical chuvoso, onde a temperatura média do mês menos quente está acima de $18{ }^{\circ} \mathrm{C}$ e o índice pluviométrico anual é relativamente elevado, porém com estação seca bem definida (BRASIL, 1982).

\section{Desenho amostral}

$\mathrm{Na}$ região norte as amostragem seguiram o protocolo de coleta do PPbio (PPBIO; SENBAM, 2013). Foram realizadas coletas em 28 parcelas (unidades amostrais) distribuídas em três módulos 
de amostragem. No primeiro módulo foram amostradas doze parcelas. No segundo e no terceiro módulos foram amostradas oito parcelas em cada. Os módulos foram distanciados aproximadamente $15 \mathrm{~km}$ entre si. Dentro dos módulos as parcelas foram distantes $1 \mathrm{~km}$ entre si. Em cada parcela foram instaladas três armadilhas pitfall distantes 50 $\mathrm{m}$ entre si, totalizando 84 armadilhas para essa região. As coletas foram realizadas entre os meses de fevereiro e março de 2011.

$\mathrm{Na}$ região sudoeste os besouros foram coletados em 22 fragmentos florestais (unidades amostrais) que variaram de 45 a 3.980 ha. Em cada fragmento foram instalados dois transectos distanciados $100 \mathrm{~m}$ entre si e a $150 \mathrm{~m}$ de qualquer borda. Em cada transecto foram instaladas três armadilhas pitfall equidistantes $50 \mathrm{~m}$, totalizando seis armadilhas por fragmento e 132 armadilhas para essa região do estado. Os dados em Tangará da Serra foram coletados de dezembro de 2011 a março de 2012. As coletas nos demais municípios da região sudoeste ocorreram entre janeiro e fevereiro de 2013.

\section{Coleta dos besouros rola-bostas}

As armadilhas pitfall foram constituídas de recipiente plástico $(19 \mathrm{~cm}$ de diâmetro e $11 \mathrm{~cm}$ de profundidade) e sobre esta foi colocada uma tampa para a proteção contra a chuva. Para atrair os besouros rola-bostas foram utilizadas aproximadamente 20 gramas de fezes humanas. As armadilhas ficaram expostas por 48 horas. Para a conservação dos insetos foi utilizado $250 \mathrm{~mL}$ de solução salina e detergente por armadilha.

Para identificação dos Scarabaeinae em nível de gênero foi utilizada a chave proposta por Vaz-de-Mello et al. (2011). Posteriormente, foram identificados a nível específico e depositados na coleção do Setor de Entomologia da Coleção Zoológica da Universidade Federal de Mato Grosso (CEMT), em Cuiabá - MT.

\section{Coleta do solo}

Em cada unidade amostral foi realizada coleta composta de solo. Na região norte, as amostras compostas foram constituídas por cinco subamostras ao longo da parcela de $250 \mathrm{~m}$ e as amostras foram coletadas a $10 \mathrm{~cm}$ do horizonte do solo. $\mathrm{Na}$ região sudoeste as amostras foram constituídas de seis subamostras retiradas exatamente do local de instalação das armadilhas pitfall. As subamostras foram homogeneizadas e posteriormente encaminhadas para análise laboratorial para determinação granulométrica. Nesse trabalho foi utilizada apenas a porção de argila, devido ao alto índice de correlação entre argila, silte e areia.

\section{Análise dos dados}

Para determinar as guildas dos besouros rola-bostas foi utilizada a classificação proposta por Cambefort (1991), onde define os gêneros de paracoprídeos e telecoprídeos e duas categorias de tamanho: pequenos $(<1 \mathrm{~cm})$ e grandes $(\geq 1 \mathrm{~cm})$. Foi Inserido nesta classificação a guilda dos endocoprídeos a qual não foi abordada originalmente por Cambefort (1991). O gênero Uroxys é considerado cleptoparasita, porém várias espécies podem ser paracoprídeos ou endocoprídeos (YOUNG, 2009), por este motivo este gênero foi removido das análises.

Para analisar o efeito da proporção de argila no solo na riqueza e na abundância dos besouros rola-bostas foram utilizados modelos lineares generalizados (GLMs) com distribuição de Poisson. Devido a superdispersão dos resíduos dos modelos foi utilizado a correção de quase-Poisson. Para todos os modelos foram utilizados dados da proporção da riqueza e da abundância de cada guilda por local. Para melhor compressão dos efeitos da granulometria do solo nas duas diferentes regiões foram realizadas analises separadamente para cada região, devido a diferença na variação da concentração de argila entre elas. Todas as análises foram feitas utilizando-se $\mathrm{o}$ software $\mathrm{R}$ (R DEVELOPMENT CORE TEAM, 2013).

\section{RESULTADOS}

As análises foram feitas com o total de 28.951 indivíduos de besouros rola-bostas. Para a região de norte os paracoprídeos grandes apresentaram a maior abundância (2.406 indivíduos), seguidos de endocoprídeos grandes (725 indivíduos) e telecoprídeos grandes (550 indivíduos). Para a região sudoeste a maior abundância foi de paracoprídeos pequenos $(9.450$ indivíduos) seguidos de endocoprídeos grandes (4.951 indivíduos) e paracoprídeos grandes (4.750 indivíduos). Ambas as regiões estudadas apresentaram maior riqueza de paracoprídeos seguida de telecoprídeos e endocoprídeos (Tabela $1)$. 
Tabela 1. Riqueza, abundância e porcentagem das guildas funcionais de besouros rola-bostas em florestas semideciduais nas regiões sudoeste e norte do estado de Mato Grosso. $\mathrm{n}=$ número de espécies/indivíduos.

\begin{tabular}{|c|c|c|c|c|c|c|c|}
\hline & Guildas & Sudoes & & Norte & & & tal \\
\hline & & $\mathrm{n}$ & $(\%)$ & $\mathrm{n}$ & $(\%)$ & $\mathrm{n}$ & $(\%)$ \\
\hline & Paracoprídeos grandes & 30 & 31,91 & 18 & 28,13 & 37 & 28,24 \\
\hline & Paracoprídeos pequenos & 29 & 30,85 & 18 & 28,13 & 42 & 32,06 \\
\hline กี & Telecoprídeos grandes & 4 & 4,26 & 4 & 6,25 & 5 & 3,82 \\
\hline$\tilde{\sigma}$ & Telecoprídeos pequenos & 17 & 18,09 & 11 & 17,19 & 26 & 19,85 \\
\hline & Endocoprídeos grandes & 9 & 9,57 & 8 & 12,50 & 13 & 9,92 \\
\hline & Endocoprídeos pequenos & 5 & 5,32 & 5 & 7,81 & 8 & 6,11 \\
\hline & Total & 94 & & 64 & & 131 & \\
\hline & Paracoprídeos grandes & 4.750 & 19,52 & 2.406 & 52,08 & 7156 & 24,72 \\
\hline & Paracoprídeos pequenos & 9.450 & 38,84 & 632 & 13,68 & 10.082 & 34,82 \\
\hline 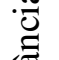 & Telecoprídeos grandes & 256 & 1,05 & 550 & 11,90 & 806 & 2,78 \\
\hline 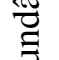 & Telecoprídeos pequenos & 2.734 & 11,24 & 236 & 5,11 & 2.970 & 10,26 \\
\hline$\frac{\bar{Z}}{\gtrless}$ & Endocoprídeos grandes & 4.951 & 20,35 & 725 & 15,69 & 5.676 & 19,61 \\
\hline & Endocoprídeos pequenos & 2.190 & 9,00 & 71 & 1,54 & 2.261 & 7,81 \\
\hline & Total & 24.331 & & 4.620 & & 28.951 & \\
\hline
\end{tabular}

\section{Efeito da granulometria sobre as guildas funcionais}

O aumento da proporção de argila no solo influenciou a estrutura das guildas funcionais dos besouros rola-bostas com efeitos distintos para as duas regiões estudadas. Para região norte o aumento da proporção de argila causou redução na riqueza de paracoprídeos grandes $(\mathrm{P}=0,03)$ e telecoprídeos grandes $(\mathrm{P}=0,10)$, e influenciou no aumento da riqueza de paracoprídeos pequenos $(\mathrm{P}=0,20)$ e na redução de endocoprídeos pequenos $(\mathrm{P}=0,12)$ e telecoprídeos grandes $(\mathrm{P}=0,12)$. Para a abundância $\mathrm{o}$ aumento da concentração de argila causou a redução de endocoprídeos pequenos $(\mathrm{P}<0,01)$ e telecoprídeos pequenos $(\mathrm{P}=0,08)$ e aumento dos endocoprídeos grandes $(\mathrm{P}=0,12)$ (Figura 2$)$.

$\mathrm{Na}$ a região sudoeste $\mathrm{o}$ aumento da proporção de argila no solo influenciou apenas no aumento da riqueza de telecoprídeos pequenos $(\mathrm{P}=$ 0,08). Quanto a abundância, o aumento na proporção de argila influenciou na redução de paracoprídeos pequenos $(\mathrm{P}=0,07)$ e aumento de telecoprídeos pequenos $(\mathrm{P}=0,03)$ e endocoprídeos grandes $(\mathrm{P}=0,10)$. $\mathrm{O}$ aumento da proporção de argila também apresentou efeito, porém com menor influência, na redução de paracoprídeos grandes $(\mathrm{P}$ $=0,21)$ (Figura 3 ). 

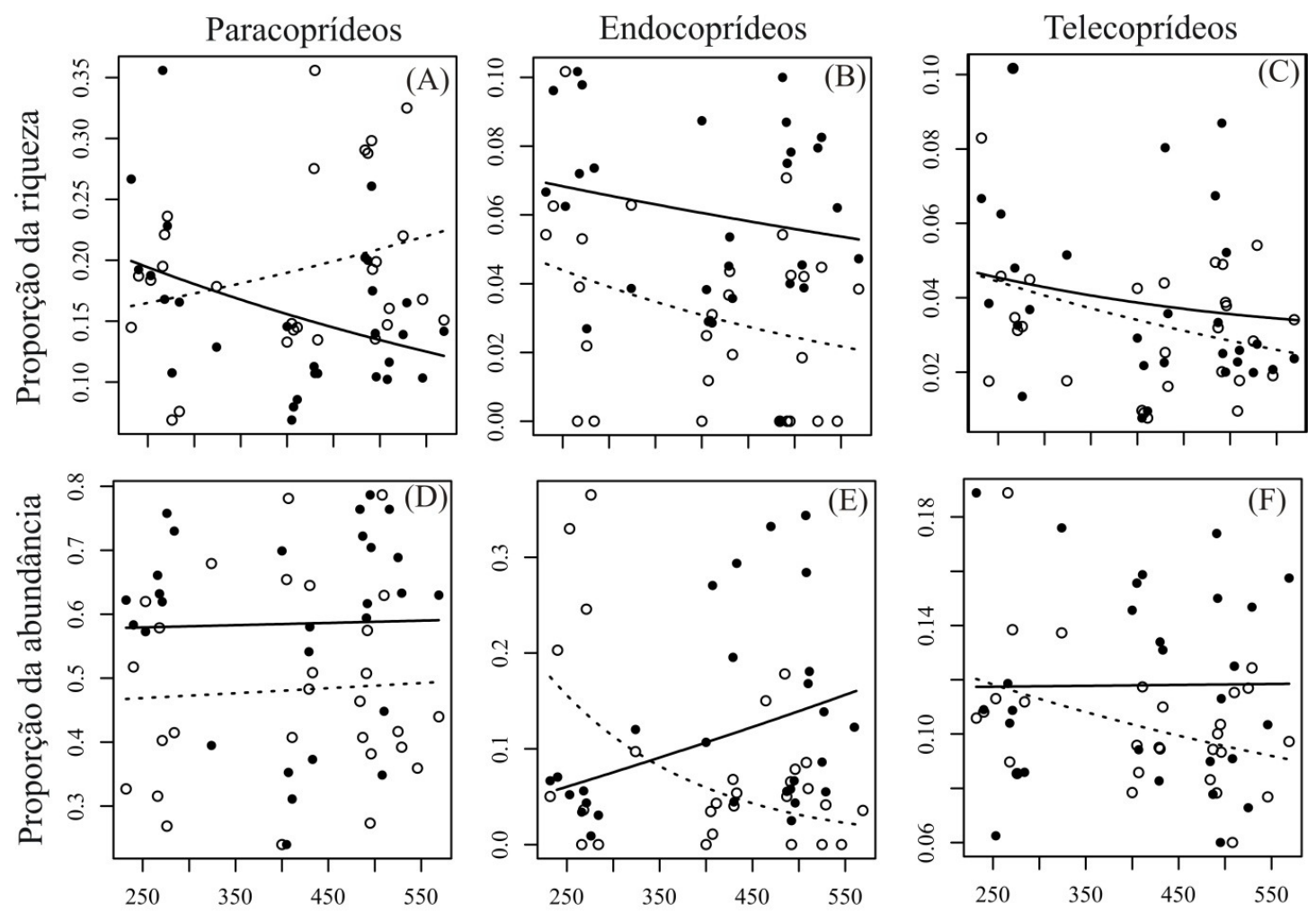

Argila

Figura 2. Efeito da concentração de argila no solo $\left(\mathrm{g} \cdot \mathrm{kg}^{-1}\right)$ na proporção riqueza e abundância das guildas dos besouros rola-bostas em florestas semideciduais na região norte do estado de Mato Grosso. Riqueza: Paracoprídeos (A); Endocoprídeos (B) e Telecoprídeos (C). Abundância : Paracoprídeos (D); Endocoprídeos (E) e Telecoprídeos (F). Círculos abertos e linha tracejada = espécies pequenas $(<1$ $\mathrm{cm})$; círculos fechados e linha contínua = espécies grandes $(\geq 1 \mathrm{~cm})$.
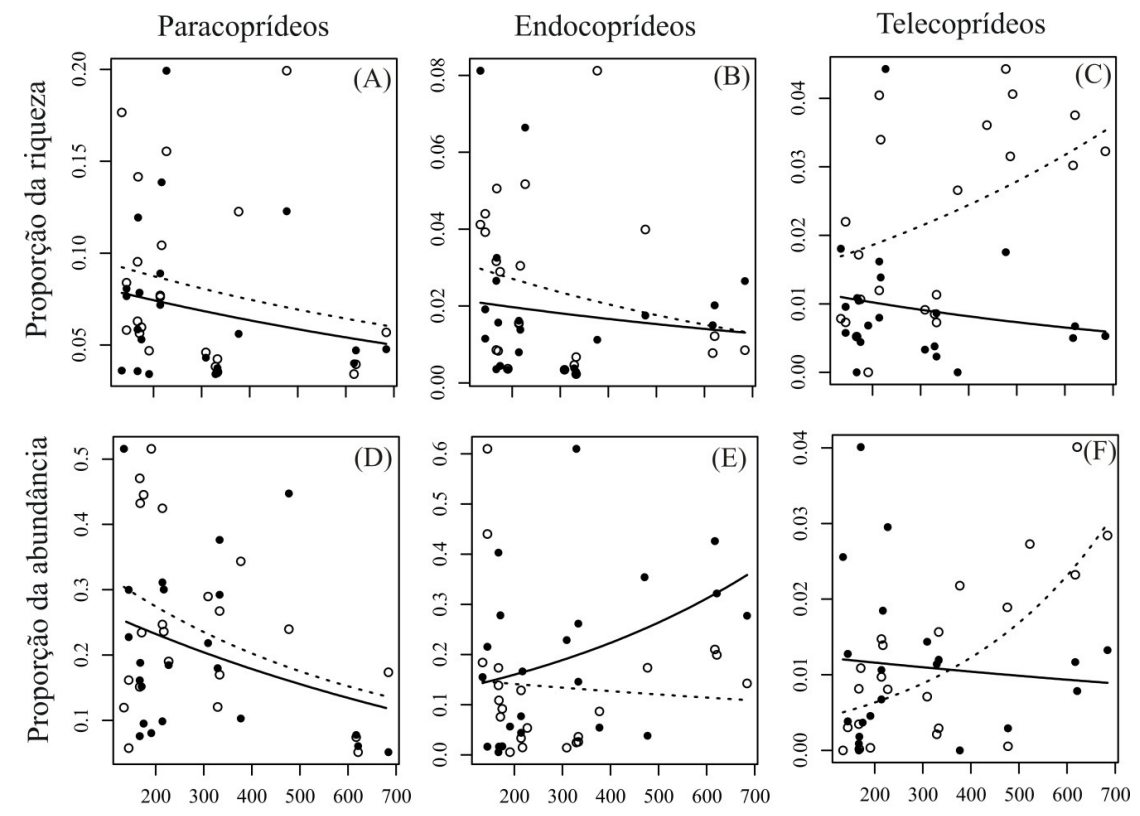

Argila

Figura 3. Efeito da concentração de argila no solo $\left(\mathrm{g} \cdot \mathrm{kg}^{-1}\right)$ na proporção riqueza e abundância das guildas dos besouros rola-bostas em florestas semideciduais na região sudoeste do estado de Mato Grosso. Riqueza : Paracoprídeos (A); Endocoprídeos (B) e Telecoprídeos (C). Abundância: Paracoprídeos (D); Endocoprídeos (E) e Telecoprídeos (F). Círculos abertos e linha tracejada = espécies pequenas $(<$ $1 \mathrm{~cm})$; círculos fechados e linha contínua = espécies grandes $(\geq 1 \mathrm{~cm})$. 


\section{DISCUSSÃO}

\section{Padrão geral das guildas funcionais:}

A maior proporção de paracoprídeos também foi encontrada em outros trabalhos para a região Neotropical (HALFFTER et al., 1992; LOUZADA; LOPES, 1997; SILVA; DI MARE, 2012). Dois processos podem explicar a dominância de paracoprídeos. O primeiro processo segue a hipótese proposta por Louzada e Lopes (1997) de que subamostras de uma comunidade de rola-bostas com distribuição geográfica maior trazem em si um determinismo taxonômico e funcional. Assim, as coletas realizadas seriam subamostras de uma região maior, a Neotropical, refletindo as proporções da comunidade como um todo. $\mathrm{O}$ segundo processo, que está atrelado ao primeiro, é o fato dos paracoprídeos apresentarem diferentes arquiteturas de ninho o que lhes permitem utilizarem a mesma massa fecal de maneira distinta. Esses besouros são capazes de construir túneis em baixo ou do lado do recurso fecal com galerias profundas e ramificadas com uma ou diversas câmaras de oviposição (HALFFTER; MATTHEWS, 1966; HALFFTER, 1977). Essas variações no uso do solo e do recurso fecal reduzem a competição entre os paracoprídeos permitindo a co-ocorrência das espécies de paracoprídeos no mesmo local (HALFFTER; EDMONDS, 1982). Aparentemente a competição entre os telecoprídeos é maior, o que causa uma redução no número de espécies locais (HANSKI; CAMBEFORT, 1991). Esses fatores podem culminar em maior proporção de espécies de paracoprídeos em escala regional (LOUZADA; LOPES, 1997).

\section{Efeito da granulometria sobre as guildas funcionais}

Em escala regional, com análise para as duas área de estudo, foi observada uma estrutura funcional dos besouros rola-bostas similar ao padrão Neotropical, com ampla dominância de paracoprídeos. Entretanto, em escala local, é possível observar que, apesar da estrutura básica se manter, existem variações significativas na riqueza e abundância em função das variações da concentração de argila no solo. Apesar da estruturação básica das guildas ser determinística em escala regional (LOUZADA; LOPES, 1997; VIDAURRE-SANCHEZ, 2011) a diversidade local (riqueza e abundância) é influenciada pelas variações da proporção de argila no solo. Isto demonstra que as condições pontuais ou locais que formam o micro-habitat são importantes para a estrutura das guildas funcionais dos besouros rolabostas somente nesta escala.

A região sudoeste apresentou maior variação na proporção de argila e os pontos de coleta estão distribuídos em maior escala geográfica. Isso torna os dados dessa região com maior poder de explicação dos efeitos da concentração de argila no solo sobre a estrutura das guildas funcionais dos besouros rola-bostas. A região norte apresentou menor variação na proporção de argila e todos os pontos estão sobre um único tipo de classificação de solo e apresentaram menor distribuição geográfica. Por isso os dados dessa região possuem menor poder de extrapolação que os dados do sudoeste do estado.

Para ambas as regiões estudadas, ocorreram alternância na abundância entre paracoprídeos e telecoprídeos pequenos. Para a região sudoeste, a redução na abundância de paracoprídeos pequenos e aumento da abundância de telecoprídeos pequenos com o aumento da concentração de argila no solo demonstra essa interação entre estas guildas. Os besouros paracoprídeos são capazes de fazer profundas galerias para alocar as fezes, utilizando-as para fazer a bola-ninho (HALFFTER; MATTHEWS, 1966). Com o aumento da concentração de argila o solo torna-se mais suscetível à compactação (SANTOS et al., 2005), o que dificulta o revolvimento do solo para confecção de grandes túneis e galerias (DOUBE, 1991). A redução da abundância dos paracoprídeos em solos com maior proporção de argila demonstra essa influência direta do tipo de solo sobre a composição das espécies da assembleia local para região sudoeste do estado de Mato Grosso.

Doube (1991) encontrou maior abundância de grandes telecoprídeos e paracoprídeos rápidos em solos com maior concentração de areia. Esse autor também afirma que na região do Kalahari, extremo Sul da África, há maior abundância de grandes telecoprídeos em solos mais arenosos. Os resultados encontrados neste estudo para a região sudoeste demonstraram que locais com solos mais arenosos apresentam maior abundância de paracoprídeos, diferindo dos resultados obtidos por Doube (1991). A compactação do solo, causado pela elevação na proporção de argila, e a redução do número de paracoprídeos nesses solos podem ter influenciado na elevação do número de telecoprídeos que não precisam escavar galerias profundas para evitar a competição. Esses besouros rolam a massa fecal para longe do local original e as enterram em galerias rasas para serem utilizadas como bolasninho para as suas proles (HALFTTER; EDMONTS, 1982). 
Os endocoprídeos não escavam galerias para alocar o recurso, por isso são menos influenciados pelo tipo de solo. $\mathrm{O}$ aumento da abundância de endocoprídeos grandes em ambas as regiões com o aumento da concentração de argila pode estar relacionado ao menor sucesso dos paracoprídeos grandes em área com maior dificuldade para construção de ninhos. Esta dificuldade leva a redução da abundância de paracoprídeos grandes e disponibiliza mais recurso para os endocoprídeos grandes. Em locais com maior dificuldade para escavação os competidores superiores, como telecoprídeos grandes e paracoprídeos grandes e rápidos, tendem a serem menos dominantes. Enquanto competidores menos eficientes, como os endocoprídeos, tendem a aumentar em solos mais compactados com maior grau de dificuldade de escavação (HANSKI; CAMBEFORT, 1991). Para a região do pantanal mato-grossense, município de Poconé, onde o solo é encharcado e apresenta dificuldade para construção de ninhos os endocoprídeos também foram os mais abundantes com dominância numérica de Trichillum externepunctatum Borre, 1886 (TISSIANI, 2009). Os Endocoprídeos pequenos como Eutrichillum, Genieridium e Trichillum apresentam comportamento de nidificação diretamente na massa fecal sem a formação de bola-ninho (ALARCÓN et al., 2009). Este comportamento difere dos demais endocoprídeos, gênero Eurysternus, que formam bolas-ninho diretamente na massa fecal (HALFFTER; EDMONDS, 1982). A redução da abundância de endocoprídeos pequenos, principalmente na região norte, não pode ser explicada pelo aumento da proporção de argila no solo, pois esse grupo de besouros possui pouca relação com o tipo de solo.

\section{CONCLUSÕES}

As guildas dos besouros rola-bostas para as regiões norte e sudoeste do estado de Mato Grosso apresentam estrutura semelhante à encontrada para a região Neotropical.

A concentração de argila no solo influenciou a estrutura das guildas funcionais apenas em nível de micro-habitat. As guildas dos paracoprídeos e telecoprídeos pequenos foram as mais afetadas pela variação da granulometria do solo.

\section{AGRADECIMENTOS}

Os autores agradecem a Fundação de Amparo à Pesquisa do Estado de Mato Grosso (FAPEMAT- 756935/2011) e o Conselho Nacional de Desenvolvimento Científico e Tecnológico (CNPq - PQ 304925/2010-1, 302997/2013-0, 405697/2013-9, 484035/2013-4, 202327/2013-2) pelo apoio financeiro; ao Programa de Pesquisa em Biodiversidade (PPbio) e a Universidade Federal de Mato Grosso pelo apoio para obtenção dos dados; ao Jorge L. Silva, Ana Silvia Tissiani, Rafael V. Nunes e aos revisores anônimos pelas contribuições no manuscrito.

\footnotetext{
ABSTRACT: This study aimed to describe the structure functional guilds of dung beetles (Scarabaeidae: Scarabaeinae) and analyze the effects of the concentration of clay in soil in this structure. The studies were developed in the north and southwest in the state of Mato Grosso. The dung beetles and soil samples were collected in the north (PPBio) and southwest (22 farms reserve) in Mato Grosso. The dung beetle were captured with pitfall traps baited with human feces exposed for 48 hours. In the north region were utilized 85 traps and 132 in the southwest. Both regions, the results showed similarity at guild structure, with paracoprids dominance, followed by telecoprids and endocoprids. Which demonstrates consistency with the Neotropical pattern. Which emphasizes the theory of the existence of a deterministic taxonomic and functional structure for the subsamples in this region. The concentration of clay showed different effects for both regions studied. To the north caused the reduction in the abundance of endocoprids and small telecoprids and reduced the richness of large paracoprids. In the southwest region had mainly influences on reducing of small paracoprids, the increase of small telecoprids and also the large endocoprids. In micro-habitat scale, concentration of clay in the soil showed an important structural element of the functional guilds of dung beetles. The Guilds of the small paracoprids and small telecoprids were most affected by the variation of soil granulometry.
}

KEYWORDS: Soil Amazon. Inter and intraspecific competition. Copro-necrophagous beetle. Scarabeids. 


\section{REFERÊNCIAS}

ALARCÓN, D. L.; HALFFTER, G.; VAZ-DE-MELLO, F. Z. Nesting Behavior in Trichillum Harold, 1868 and related genera (Coleoptera: Scarabaeidae: Scarabaeinae: Ateuchini: Scatimina): a primitive process or a loss of nidification? The Coleopterists Bulletin, v. 63, p. 289-297, 2009. http://dx.doi.org/10.1649/1132.1

BRASIL. MINISTÉRIO DAS MINAS E ENERGIA. SECRETARIA GERAL. Projeto RADAMBRASIL: Folha SF. 21 Cuiabá; geologia, geomorfologia, pedologia, vegetação e uso potencial da terra. Rio de Janeiro, 1982. $544 \mathrm{p}$.

CAMBEFORT, Y. Biogeography and evolution. In: HANSKI, I.; CAMBEFORT, Y. (Ed.) Dung beetle ecology. Cambridge: Princeton University Press, 1991. p. 52-67, 1991. http://dx.doi.org/10.1515/9781400862092.51

DOUBE, B. M. A functional classification for analysis of the structure of dung beetle assemblages. Ecological Entomology. v. 15, p. 371-383, 1990. http://dx.doi.org/10.1111/j.1365-2311.1990.tb00820.x

DOUBE, B. M. Dung Beetles of Southern Africa. In: HANSKI, I.; CAMBEFORT, Y. (Ed.) Dung beetle ecology. Cambridge: Princeton University Press, $1991 . \quad$ p. 133-155. http://dx.doi.org/10.1515/9781400862092.133

FILGUEIRAS, B. K.C.; IANNUZZI, L.; LEAL, I. R. Habitat fragmentation alters the structure of dung beetle communities in the Atlantic Forest. Biological Conservation. v. 144, p. 362-369, 2011. http://dx.doi.org/10.1016/j.biocon.2010.09.013

HALFFTER, G. Evolution of nidification in the Scarabaeinae (Coleoptera, Scarabaeidae). Quaestiones Entomologicae. v. 13, p. 231-253, 1977.

HALFFTER, G.; EDMONDS, W. D. The nesting behavior of dung beetles (Scarabaeinae): an ecological and evolutive approach. Unesco, Instituto Ecologia, México. p. 155, 1982.

HALFFTER, G.; FAVILA, M. E.; HALFFTER, V. A comparative study of the structure of the scarab guild in American tropical rain forests and derived ecosystems. Folia Entomologica Mexicana. n. 84, p. 131-156, 1992.

HALFFTER, G.; MATTHEWS, E. G. The natural history of dung beetles of the subfamily Scarabaeinae (Coleoptera, Scarabaeidae). Folia Entomologica Mexicana, v. 12, p. 1-312, 1966.

HANSKI, I.; CAMBEFORT, Y. Competition in Dung Beetles. In: HANSKI, I.; CAMBEFORT, Y. (Ed.) Dung beetle ecology. Cambridge: Princeton University Press, 1991. p. 305-329. http://dx.doi.org/10.1515/9781400862092.305 http://dx.doi.org/10.1515/9781400862092

LOUZADA, J. N. C.; LOPES, F. S. A comunidade de Scarabaeidae copro-necrófago (Coleoptera) de um fragmento de Mata Atlântica. Revista brasileira de Entomologia, v. 41, n. 1, p. 117-121, 1997.

LUMARET, J. P,; KIRK, A. A. Ecology of Dung beetles in the French Mediterranean region. Acta Zoologica Mexicana. v. 24, p. 1-55, 1987.

PHILIPS, T. K. The evolution history and diversification of dung beetles. In: SIMMONS, L. W.; RIDSDILL-SMITH, T.J. (Ed.) Ecology and evolution of dung beetles. Oxford: Wiley-Blackwell Publishing Ltd, 2011. p. 21-45. http://dx.doi.org/10.1002/9781444342000.ch2

PPBIO; CENBAM. Sinop. Disponível em: <http://ppbio.inpa.gov.br/sitios/sinop>. Acesso em: 28 jul. 2013. 
R DEVELOPMENT CORE TEAM. R. A language and environment for statistical computing. R Foundation for Statistical Computing, Vienna, Austria. Disponível em: http://www.R-project.org/.

SÁNCHEZ, R. O. Zoneamento agroecológico do Estado de Mato Grosso: ordenamento ecológicopaisagístico do meio. Cuiabá: Fundação de Pesquisas Cândido Rondon, 1992. 160 p.

VIDAURRE-SANCHEZ, T. Convergência ecológica de comunidades de Scarabaeinae m sistemas nativos e pastagens introduzidas da Américas do Sul. 2011. 100f. Dissertação (Mestrado em Ecologia Aplicada) Universidade Federal de Lavras, Lavras, 2011.

SANTOS, R. D.; LEMOS, R. C.; SNATOS, H. G.; KER, J. C.; ANJOS, L. H. C. Manual de descrição de coleta de solo no campo. 5. ed. Viçosa: Folha de Viçosa, 2005. 52 p.

SEPLAN - Secretaria de estado de planejamento e coordenação geral. Mapa A001:Mapa de solos do estado de Mato Grosso. Mato Grosso, 2001. Mapa. Escala 1:1500000.

SILVA, P. G.; AUDINO, L. D.; NOGUEIRA, J. M.; MORAES, L. P.; VAZ DE MELLO, F. Z. Escarabeineos (Coleoptera: Scarabaeidae: Scarabaeinae) de uma área de campo nativo no bioma Pampa, Rio Grande do Sul, Brazil. Biota Neotropical, v. 12, n. 3, p. 1-9, 2012. http://dx.doi.org/10.1590/S1676-06032012000300024

SILVA, P. G.; DI MARE, R. Escarabeíneos copro-necrófagos (Coleoptera, Scarabaeidae, Scarabaeinae) de fragmentos de Mata Atlântica em Silveira Martins, Rio Grande do Sul, Brasil. Iheringia, Série Zoologia, v. 102, p. 197-205, 2012. http://dx.doi.org/10.1590/S0073-47212012000200012

SILVA, P. G.; GARCIA, A. R.; VIDAL, M. B. Besouros copro-necrófagos (Coleoptera: Scarabaeidae stricto sensu) coletados em ecótono natural de campo e mata em Bagé, RS. Ciência e Natura, v. 30, n. 2, p. 71-91, 2008.

TISSIANI, A. S. O. Composição da comunidade de Scarabaeidae coprófagos (Insecta, Coleoptera), em uma área na região norte do pantanal de Mato Grosso. 2009. 88f. Dissertação (Mestrado em Ecologia e Conservação da Biodiversidade) - Universidade Federal de Mato Grosso, Cuiabá, 2009.

VAZ-DE-MELLO, F. Z.; EDMONDS, W. D.; OCAMPO, F.; SCHOOLMEESTERS, PAUL. A multilingual key to the genera and subgenera of the subfamily Scarabaeinae of the New World. Zootaxa, v. 2854, p. 1-73, 2011.

YOUNG, O. P. Observations on burrowing behavior by panamanian scarabaeinae (Coleoptera: Scarabaeidae).

The Coleopterists Bulletin, v. 63, n. 3, p. 319-324. http://dx.doi.org/10.1649/1164.1 
Anexo 1. Abundância de espécies de besouros rola-bostas (Scarabaeidae: Scarabaeinae) coletados em áreas de floresta estacional no sudoeste (SO) e no norte $(\mathrm{N})$ do Estado de Mato Grosso durante os anos de 2011, 2012 e 2013. E = Endocoprídeos; T = Telecoprídeos; $\mathrm{P}=$ Paracoprídeos; $\mathrm{G}=$ grande $(\geq 1 \mathrm{~cm})$; $\mathrm{Pe}=$ pequeno $(<1 \mathrm{~cm})$.

\begin{tabular}{|c|c|c|c|c|}
\hline Espécies & Guilda & Tamanho & Região SO & Região N \\
\hline Agamopus sp. & $\mathrm{T}$ & $\mathrm{Pe}$ & 6 & 0 \\
\hline Anomiopus sp.1 & $\mathrm{P}$ & $\mathrm{Pe}$ & 1 & 0 \\
\hline Ateuchus aff. striatulus & $\mathrm{P}$ & $\mathrm{Pe}$ & 211 & 0 \\
\hline Ateuchus aff. carbonarius & $\mathrm{P}$ & $\mathrm{Pe}$ & 191 & 0 \\
\hline Ateuchus connexus (Harold, 1868) & $\mathrm{P}$ & $\mathrm{Pe}$ & 0 & 157 \\
\hline Ateuchus sp.1 & $\mathrm{P}$ & $\mathrm{Pe}$ & 0 & 36 \\
\hline Ateuchus sp.2 & $\mathrm{P}$ & $\mathrm{Pe}$ & 0 & 1 \\
\hline Ateuchus sp.3 & $\mathrm{P}$ & $\mathrm{Pe}$ & 0 & 3 \\
\hline Ateuchus sp.4 & $\mathrm{P}$ & $\mathrm{Pe}$ & 22 & 0 \\
\hline Ateuchus sp.5 & $\mathrm{P}$ & $\mathrm{Pe}$ & 2 & 0 \\
\hline Ateuchus sp.6 & $\mathrm{P}$ & $\mathrm{Pe}$ & 2 & 0 \\
\hline Ateuchus sp.7 & $\mathrm{P}$ & $\mathrm{Pe}$ & 3.475 & 0 \\
\hline Besourenga horacioi Matínez, 1967 & $\mathrm{E}$ & $\mathrm{G}$ & 0 & 3 \\
\hline Canthidium aff. gerstaeckeri & $\mathrm{P}$ & $\mathrm{Pe}$ & 172 & 28 \\
\hline Canthidium aff. lentum & $\mathrm{P}$ & $\mathrm{Pe}$ & 0 & 40 \\
\hline Canthidium aff. pinotoides & $\mathrm{P}$ & $\mathrm{Pe}$ & 481 & 66 \\
\hline Canthidium dohrni Harold, 1867 & $\mathrm{P}$ & $\mathrm{Pe}$ & 0 & 3 \\
\hline Canthidium sp.1 & $\mathrm{P}$ & $\mathrm{Pe}$ & 0 & 1 \\
\hline Canthidium sp.2 & $\mathrm{P}$ & $\mathrm{Pe}$ & 1.177 & 0 \\
\hline Canthidium sp.3 & $\mathrm{P}$ & $\mathrm{Pe}$ & 8 & 0 \\
\hline Canthidium sp.4 & $\mathrm{P}$ & $\mathrm{Pe}$ & 93 & 0 \\
\hline Canthidium sp.5 & $\mathrm{P}$ & $\mathrm{Pe}$ & 3 & 0 \\
\hline Canthidium sp.6 & $\mathrm{P}$ & $\mathrm{Pe}$ & 11 & 0 \\
\hline Canthidium sp.7 & $\mathrm{P}$ & $\mathrm{Pe}$ & 31 & 0 \\
\hline Canthidium sp.8 & $\mathrm{P}$ & $\mathrm{Pe}$ & 427 & 0 \\
\hline Canthidium sp.9 & $\mathrm{P}$ & $\mathrm{Pe}$ & 2 & 0 \\
\hline Canthidium sp.10 & $\mathrm{P}$ & $\mathrm{Pe}$ & 16 & 0 \\
\hline Canthidium sp.11 & $\mathrm{P}$ & $\mathrm{Pe}$ & 0 & 6 \\
\hline Canthidium sp.12 & $\mathrm{P}$ & $\mathrm{Pe}$ & 0 & 3 \\
\hline Canthidium sp.13 & $\mathrm{P}$ & $\mathrm{Pe}$ & 0 & 5 \\
\hline Canthidium sp.14 & $\mathrm{P}$ & $\mathrm{Pe}$ & 0 & 2 \\
\hline Canthidium sp.15 & $\mathrm{P}$ & $\mathrm{Pe}$ & 0 & 1 \\
\hline Canthidium sp.16 & $\mathrm{P}$ & $\mathrm{Pe}$ & 0 & 10 \\
\hline Canthidium sp.17 & $\mathrm{P}$ & $\mathrm{Pe}$ & 12 & 0 \\
\hline Canthidium sp.18 & $\mathrm{P}$ & $\mathrm{Pe}$ & 2 & 0 \\
\hline Canthon aff. chalybaeum & $\mathrm{T}$ & $\mathrm{Pe}$ & 79 & 19 \\
\hline Canthon aff. chiriguano & $\mathrm{T}$ & $\mathrm{Pe}$ & 51 & 0 \\
\hline Canthon aff. fulgidum & $\mathrm{T}$ & $\mathrm{Pe}$ & 0 & 17 \\
\hline Canthon aff. scrutator & $\mathrm{T}$ & $\mathrm{Pe}$ & 39 & 0 \\
\hline Canthon aff. sericatus & $\mathrm{T}$ & $\mathrm{Pe}$ & 0 & 8 \\
\hline Canthon bimaculatus Schmidt, 1922 & $\mathrm{~T}$ & $\mathrm{Pe}$ & 390 & 0 \\
\hline Canthon brunneus Schmidt, 1922 & $\mathrm{~T}$ & $\mathrm{Pe}$ & 197 & 0 \\
\hline Canthon aff. triangularis & $\mathrm{T}$ & $\mathrm{Pe}$ & 743 & 0 \\
\hline Canthon aff. coloratus & $\mathrm{T}$ & $\mathrm{Pe}$ & 4 & 0 \\
\hline Canthon dives Harold, 1868 & $\mathrm{~T}$ & $\mathrm{Pe}$ & 0 & 8 \\
\hline Canthon fulgidus Redtenbacher, 1867 & $\mathrm{~T}$ & $\mathrm{Pe}$ & 0 & 13 \\
\hline Canthon histrio (Le Peletier e Serville,1828) & $\mathrm{T}$ & $\mathrm{Pe}$ & 437 & 0 \\
\hline Canthon proseni Marínez, 1949 & $\mathrm{~T}$ & $\mathrm{Pe}$ & 0 & 1 \\
\hline Canthon quinquemaculatus Castelnau, 1840 & $\mathrm{~T}$ & $\mathrm{Pe}$ & 24 & 2 \\
\hline
\end{tabular}


Canthon rufocoeruleus Martínez, 1948

Canthon triangularis Drury, 1770

Canthonella sp.1

Coprophanaeus cyanescens Olsoufieff, 1924

Coprophanaeus degallieri Arnaud, 1997

Coprophanaeus ensifer Germar, 1821

Coprophanaeus jasius (Olivier, 1789)

Coprophanaeus spitzi (Pessôa, 1934)

Coprophanaeus telamon (Linnaeus, 1767)

Coprophanaeus terrali Arnaud, 2002

Cryptocanthon campbellorum Howden, 1973

Deltochilum amazonicum Bates, 1887

Deltochilum carinatum (Westwood, 1837)

Deltochilum enceladum Kolbe, 1893

Deltochilum orbiculare Lansberge, 1874

Deltochilum sp.1

Deltochilum sp.2

Deltochilum sp.3

Dichotomius aff. semiaeneus

Dichotomius aff. lucasi

Dichotomius aff. lucasi 2

Dichotomius bos (Blanchard, 1846)

SILVA, R. J. et al

Dichotomius carbonarius (Mannerheim, 1929)

Dichotomius carinatus (Luederwaldt, 1925)

Dichotomius cuprinus (Felsche, 1901)

Dichotomius globulus (Felsche, 1901)

Dichotomius aff. imitator

Dichotomius lycas (Felsche, 1901)

Dichotomius mamillatus (Felsche, 1901)

Dichotomius melzeri (Luederwaldt, 1922)

Dichotomius nimuendaju Luederwaldt, 1925

Dichotomius nisus (Olivier, 1789)

Dichotomius opacipennis (Luederwaldt, 1931)

Dichotomius sp.1

Dichotomius worontzowi (Pereira, 1942)

Eurysternus atrosericus Génier, 2009

Eurysternus caribaeus (Herbst 1789)

Eurysternus cyclops Génier, 2009

Eurysternus foedus Guérin-Méneville, 1844

Eurysternus hamaticollis Balthasar 1939

Eurysternus harlequin Génier 2009

Eurysternus howdeni Génier, 2009

Eurysternus hypocrita Balthasar, 1939

Eurysternus jessopi Martínez, 1988

Eurysternus plebejus Harold, 1880

Eurysternus uniformis Génier 2009

Eurysternus vastiorum Martínez, 1988

Eurysternus wittmerorum Martínez, 1988

Eutrichillum sp.1

Eutrichillum sp.2

Eutrichillum sp.3

Genieridium bidens (Balthasar, 1942)

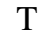

$\mathrm{T}$

$\mathrm{T}$

$P$

$\mathrm{P}$

$\mathrm{P}$

$P$

$P$

$\mathrm{P}$

$\mathrm{P}$

$\mathrm{T}$

T

$\mathrm{T}$

$\mathrm{T}$

$\mathrm{T}$

$\mathrm{T}$

$\mathrm{T}$

T

$\mathrm{P}$

$\mathrm{P}$

$\mathrm{P}$

$\mathrm{P}$

$\mathrm{P}$

P

$\mathrm{P}$

P

$\mathrm{P}$

P

P

$\mathrm{P}$

P

P

P

$\mathrm{P}$

$\mathrm{P}$

E

E

E

E

E

E

E

E

E

E

E

E

E

E

E

E

E

E
$\mathrm{Pe}$

$\mathrm{Pe}$

$\mathrm{Pe}$

G

G

G

G

G

G

G

$\mathrm{Pe}$

G

G

G

G

G

G

G

G

G

G

G

G

G

G

G

G

G

G

G

G

G

G

G

G

$\mathrm{G}$
$\mathrm{Pe}$

G

G

G

G

G

G

G

G

G

G

G

G

Pe

$\mathrm{Pe}$

$\mathrm{Pe}$
$\mathrm{Pe}$

$\mathrm{Pe}$
0

94

0

0

17

112

2

0

0

4

1

17

7

6

520

71

0

0

0

815

0

0

0

18

0

1030

28

0

15

42

0

4

0

0

17

60

79

7

4

3

0

0

32

5

0

0

1

594

1

2

0

0 
SILVA, R. J. et al

Genieridium cryptops (Arrow, 1913)

Malagoniella aff. punctatostriata

Ontherus appendiculatus (Mannerheim, 1829)

Ontherus azteca Harold, 1869

Ontherus digitatus Harold, 1868

Ontherus planus Génier, 1996

Ontherus sp. 1

Ontherus sp.2

Onthophagus sp.1

Onthophagus sp.2

Onthophagus aff. hirculus

Onthophagus aff. buculus

Onthophagus digitifer Boucomont, 1932

Onthophagus aff. haematopus

Onthophagus onthochromus Arrow, 1913

Oxysternon conspicillatum Weber, 1801

Oxysternon macleayi Nevison, 1892

Oxysternon palemo Castelnau, 1840

Oxysternon silenus (Laporte, 1840)

Phanaeus sororibispinus Edmonds \& Zidek, 2012

Phanaeus cambeforti Arnaud, 1982

Phanaeus chalcomelas (Perty, 1830)

Phanaeus palaeno Blanchard, 1843

Scybalocanthon uniplagiatus (Schmidt, 1922)

Scybalocanthon aereus (Schmidt, 1922)

Sulcoplanaeus faunus (Fabricius, 1775)

Sylvicanthon sp. 1

Sylvicanthon sp.2

Trichillum externepunctatum Borre, 1886

Trichillum sp.1

Total

\begin{tabular}{|c|c|c|c|}
\hline E & $\mathrm{Pe}$ & 127 & 0 \\
\hline $\mathrm{T}$ & G & 4 & 0 \\
\hline$P$ & $\mathrm{Pe}$ & 32 & 0 \\
\hline$P$ & $\mathrm{G}$ & 36 & 0 \\
\hline$P$ & $\mathrm{Pe}$ & 75 & 0 \\
\hline $\mathrm{P}$ & $\mathrm{G}$ & 1 & 0 \\
\hline$P$ & $\mathrm{G}$ & 0 & 6 \\
\hline$P$ & $\mathrm{G}$ & 0 & 5 \\
\hline$P$ & $\mathrm{Pe}$ & 693 & 265 \\
\hline$P$ & $\mathrm{Pe}$ & 2 & 0 \\
\hline$P$ & $\mathrm{Pe}$ & 92 & 2 \\
\hline$P$ & $\mathrm{Pe}$ & 2 & 0 \\
\hline$P$ & $\mathrm{Pe}$ & 3 & 0 \\
\hline$P$ & $\mathrm{Pe}$ & 2.201 & 0 \\
\hline$P$ & $\mathrm{Pe}$ & 11 & 3 \\
\hline$P$ & $\mathrm{G}$ & 345 & 0 \\
\hline$P$ & $\mathrm{G}$ & 0 & 284 \\
\hline$P$ & $\mathrm{G}$ & 1 & 0 \\
\hline$P$ & $\mathrm{G}$ & 24 & 0 \\
\hline $\mathrm{P}$ & G & 2 & 2 \\
\hline$P$ & $\mathrm{G}$ & 36 & 0 \\
\hline$P$ & $\mathrm{G}$ & 10 & 3 \\
\hline $\mathrm{P}$ & $\mathrm{G}$ & 1 & 0 \\
\hline $\mathrm{T}$ & $\mathrm{Pe}$ & 100 & 0 \\
\hline $\mathrm{T}$ & $\mathrm{Pe}$ & 1 & 0 \\
\hline$P$ & $\mathrm{G}$ & 4 & 1 \\
\hline $\mathrm{T}$ & $\mathrm{Pe}$ & 0 & 2 \\
\hline $\mathrm{T}$ & $\mathrm{Pe}$ & 540 & 0 \\
\hline E & $\mathrm{Pe}$ & 796 & 0 \\
\hline E & $\mathrm{Pe}$ & 0 & 5 \\
\hline
\end{tabular}

